\title{
cDNA Probes for the Diagnosis of Bovine Torovirus (Breda Virus) Infection
}

\author{
M. KOOPMANS, $\dagger$ E. J. SNIJDER, $\ddagger$ AND M. C. HORZINEK* \\ Department of Infectious Diseases and Immunology, Virology Division, Veterinary Faculty, State University, Yalelaan 1, \\ 3584CL, Utrecht, The Netherlands
}

Received 30 July 1990/Accepted 17 December 1990

\begin{abstract}
A genomic cDNA library of RNA from Breda virus (BRV), a bovine torovirus, was prepared. The nucleotide sequence of the $3^{\prime}$ end of the genome was found to be highly conserved (93\% identical) between BRV and Berne virus, the torovirus prototype. Cross-hybridization experiments were performed to select Berne virus cDNA clones for use as probes in a dot hybridization assay; the objective was to detect heterologous torovirus RNA in fecal material. A rapid RNA extraction method was employed to make the test applicable for routine diagnosis. Samples from calves after experimental and natural infection with BRV were assayed to establish the sensitivity and specificity of the test and to compare the test with the enzyme-linked immunosorbent assay (ELISA) for antigen detection. For this purpose, 53 samples from seven infected calves were tested with both methods. In the ELISA, BRV was detected in six fecal samples from three inoculated calves. By use of the hybridization test, 16 samples from seven calves reacted positively. With one exception, only postinoculation samples were found positive in hybridization. No signal was seen in feces from uninoculated calves or from calves infected with rotavirus or coronavirus.
\end{abstract}

Toroviruses are enveloped, positive-stranded RNA viruses which cause enteric infections in animals and possibly in humans (4). The torovirus prototype is Berne virus (BEV), which was isolated from a horse in Berne, Switzerland. BEV can be propagated in embryonic mule skin (EMS) cells (19) and is currently the only cultivable torovirus. A cDNA library has been prepared from RNA isolated from BEV-infected EMS cells (16). Clones covering about $15 \mathrm{~kb}$ of the BEV genome [starting at its poly(A) tail] have been identified and sequenced $(13,14,16)$.

Breda viruses (BRVs) are antigenically related toroviruses that have been found in cattle $(20,21)$. BRVs cause diarrhea both in experimentally infected gnotobiotic calves (GCs) (20) and under field conditions (5). In older cattle, BRV infections have been associated with outbreaks of diarrhea and reduced milk production (9). For routine diagnosis, an enzyme-linked immunosorbent assay (ELISA) is used to detect BRV antigen and antibodies $(5,8)$. However, infected animals appear to shed ELISA-detectable amounts of virus for only 2 to 3 days (20). Also, BRV has to be purified from feces of GCs for use as an antigen in ELISA. The latter fact and the need for a more sensitive assay for virus detection have led us to explore alternatives to immunological diagnostic tests; these alternatives are based on the detection of specific nucleotide sequences instead of antigens (e.g., hybridization and polymerase chain reaction amplification).

In this report the application of a set of BEV cDNA clones as probes in a hybridization test for the diagnosis of torovirus infections in bovines is described. Sequence information from the $3^{\prime}$ end of the BRV genome is presented and compared with the corresponding BEV sequence, which has been reported previously (14).

\footnotetext{
* Corresponding author.

† Present address: Centers for Disease Control, Atlanta, GA 30333.

$\ddagger$ Present address: Department of Virology, Institute of Medical Microbiology, State University of Leiden, 2300AH, Leiden, The Netherlands.
}

\section{MATERIALS AND METHODS}

Methods of RNA isolation. The template RNA for cDNA synthesis was extracted from the feces of a BRV-infected GC (GC58) (21). The guanidinium isothiocyanate-cesium chloride method of Maniatis et al. (10) was used; this protocol is suitable for extraction of RNA from sources that are very rich in RNase and other proteins (e.g., feces). For the diagnostic hybridization assays, RNA was extracted essentially as previously described by Boom et al. (1). Briefly, a 1:20 suspension of feces in $6 \mathrm{M}$ guanidinium isothiocyanate (Fluka Chemie AG, Buchs, Switzerland) was clarified by low-speed centrifugation $(20 \mathrm{~min}$ at $2,000 \times \mathrm{g})$, and nucleic acids were adsorbed to size-fractionated silicon dioxide particles (Sigma Chemical Company, St. Louis, Mo.). Elution of the nucleic acids (RNA) was done by using TE buffer (10 mM Tris, $1 \mathrm{mM}$ EDTA [pH 8.0]) in the presence of 0.5 to $1 \mathrm{U}$ of RNasin per $\mu$ l. Intracellular poly(A)-selected RNAs from BEV-infected EMS cells and from $\mathrm{Sac}^{-}$cells, infected with mouse hepatitis virus (MHV), were used as positive and negative controls, respectively $(15,17)$.

Synthesis, cloning, and sequencing of BRV cDNA. A cDNA library of genomic BRV RNA was prepared by the method of Gubler and Hoffman (3) and oligo(dT) priming. cDNA was $\mathrm{dC}$ tailed and cloned into dG-tailed plasmid pUC9 (Pharmacia Inc., Uppsala, Sweden). Recombinant transformants were screened by colony hybridization (10) with $\alpha-{ }^{32} \mathrm{P}$ labeled BEV cDNA fragments as probes. Cross-hybridizing BRV clones were sequenced and compared with known BEV nucleotide sequences.

Selection of BEV cDNA clones. In order to select BEV sequences that cross-hybridize with BRV genomic RNA, plasmid DNA from BEV cDNA clones $(13,16)$ was denatured by adding 0.25 volumes of $1 \mathrm{M} \mathrm{NaOH}$. After $10 \mathrm{~min}$ at $65^{\circ} \mathrm{C}$, an equal volume of $2 \mathrm{M}$ ammonium acetate was added and the mixture was spotted onto nitrocellulose filters ( $\mathrm{Ny}$ tran; Schleicher \& Schuell, Dassel, Germany), which were then baked for $2 \mathrm{~h}$ at $80^{\circ} \mathrm{C}$; pUC9 DNA was spotted as a negative control. For use as a probe, genomic BRV RNA, 


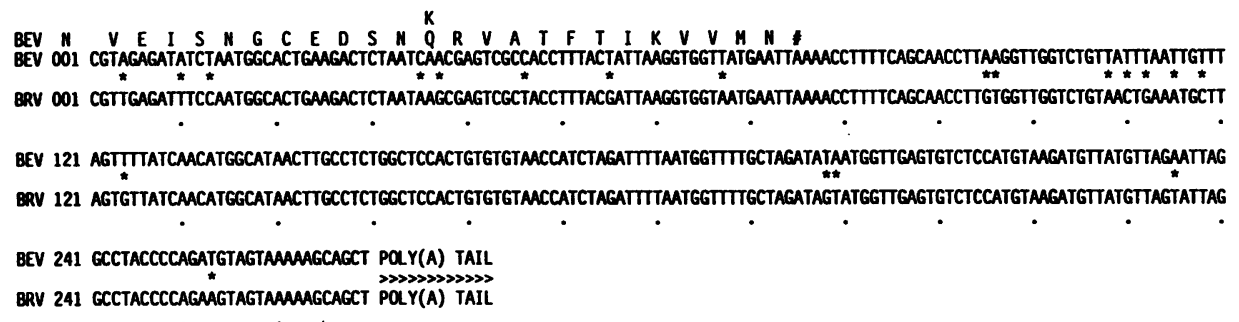

FIG. 1. Alignment of the nucleotide sequence from the $3^{\prime}$ end of the BEV genome (upper sequence) with that of BRV (lower sequence); the termination codon of the BEV N protein gene is located at nucleotide positions 74 to 76 . Nucleotide substitutions are marked with an asterisk. The predicted amino acid sequence of the $\mathrm{N}$ protein of $\mathrm{BEV}$ and a single substitution ( $\mathrm{K}$ for $\mathrm{Q}$ in $\mathrm{BRV}$ ) are listed.

extracted from GC58 fecal material, was fragmented and labeled with $\left[{ }^{32} \mathrm{P}\right] \mathrm{ATP}$ by using $\mathrm{T} 4$ polynucleotide kinase (16). Hybridization was done at $42^{\circ} \mathrm{C}$, essentially as previously described by van Berlo et al. (18).

Diagnostic hybridizations. RNA that had been extracted from $50-\mu l$ samples with the use of silicon dioxide particles was spotted in duplicate on nitrocellulose filters. After proteinase $\mathrm{K}$ digestion $\left(30 \mathrm{~min}\right.$ at $42^{\circ} \mathrm{C}$ ) $(10)$, hybridization was performed at $42^{\circ} \mathrm{C}$, essentially as previously described by van Berlo et al. (18). As probes, BEV cDNA fragments which had been labeled with $\left[\alpha-{ }^{32}\right.$ P]dATP with the use of heat denaturation, pentanucleotide primers, and the Klenow fragment of Escherichia coli polymerase I (2) were used.

ELISA, hemagglutination test, and solid-phase immune electron microscopy for detection of BRV in feces. A double antibody sandwich ELISA was used with rabbit pre- and

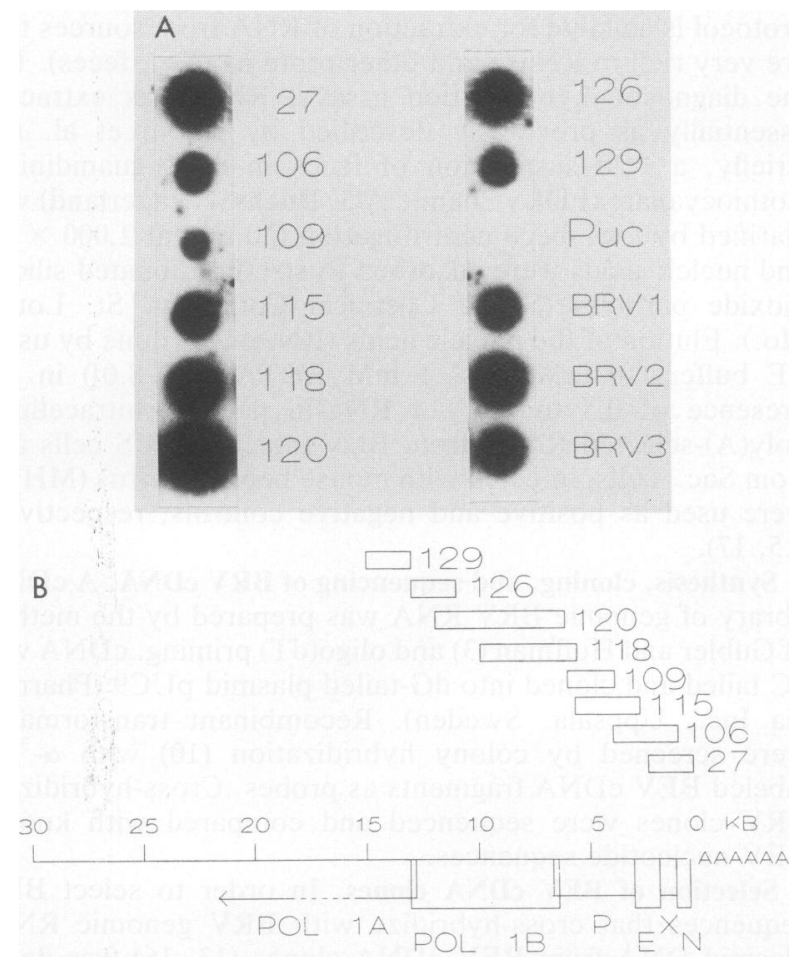

FIG. 2. (A) Hybridization of eight cDNA clones of BEV and three of BRV with BRV genomic RNA. Plasmid DNA (pUC9) was included as a negative control. (B) Schematic representation of the BEV genome and the localization of the cDNA clones. Boxed areas indicate open reading frames encoding nucleocapsid $(\mathrm{N})$, envelope $(E)$, peplomer $(\mathrm{P})$, and polymerase $(\mathrm{POL})$ proteins; the product of gene $X$ is unidentified.
post-BRV immunization immunoglobulin $\mathbf{G}$ as a capture antibody and GC76 anti-BRV immunoglobulin $G$ conjugated to horseradish peroxidase as a detecting antibody (5). The hemagglutination test for the detection of BRV by using rat erythrocytes has been previously described (20). Solid-phase immune electron microscopy was performed as previously described (5).

Propagation, concentration, and quantitation of virus. BEV was grown and titrated to a $50 \%$ tissue culture infective dose $\left(\mathrm{TCID}_{50}\right)$ in EMS cells as previously described (19). Viruscontaining culture supernatants were concentrated by using ammonium sulfate precipitation (19) and were stored at $-70^{\circ} \mathrm{C}$. The propagation of MHV has been previously described (17).

Origin of samples. A total of 53 fecal samples $(n=53)$ from seven GCs before and after oral infection with BRV, astrovirus, and cryptosporidia, as well as materials from three uninoculated calves, were kindly provided by G. N. Woode (Texas A \& M University). The samples were stored at $-20^{\circ} \mathrm{C}$ until testing. Feces from diarrheal calves reacting in routine rotavirus and coronavirus ELISAs were obtained from the Large Animal Clinic, Utrecht, The Netherlands.

Nucleotide sequence accession number. The BRV nucleotide sequence has been assigned EMBL accession no. X53536.

\section{RESULTS}

Cloning and sequencing of the $3^{\prime}$ end of the BRV genome. To assess the degree of genetic similarity between the two toroviruses, the $3^{\prime}$ end of the BRV genome was cloned, sequenced, and compared with that of BEV. A total of 384 transformants were screened, and 14 of them were found to react with BEV cDNA clone 27 , containing $2 \mathrm{~kb}$ of cDNA from the $3^{\prime}$ end of the BEV genome. Agarose gel electrophoresis of PstI-digested plasmid DNA (10) revealed that all hybridization-positive clones contained short cDNA inserts $(<300 \mathrm{bp})$. Three of these poly(A)-containing clones were sequenced, and a comparison was made with the $3^{\prime}$ end of the BEV genome (Fig. 1). The nucleotide sequences of BRV and BEV were found to be $93 \%$ identical in this area, which includes the $3^{\prime}$ end of the BEV nucleocapsid $(\mathrm{N})$ protein gene (14). Twenty-four of the 25 carboxyl-terminal amino acids of the BEV $\mathrm{N}$ protein are also encoded by one of the reading frames in the corresponding region of the BRV genome (Fig. 1).

Selection of cross-hybridizing BEV cDNA clones. When BRV genome RNA was hybridized to a set of BEV cDNA clones, signals of variable intensity were obtained, whereas pUC9 plasmid DNA did not react (Fig. 2A). Only clone 109, which contains a part of the BEV peplomer $(\mathrm{P})$ protein gene 

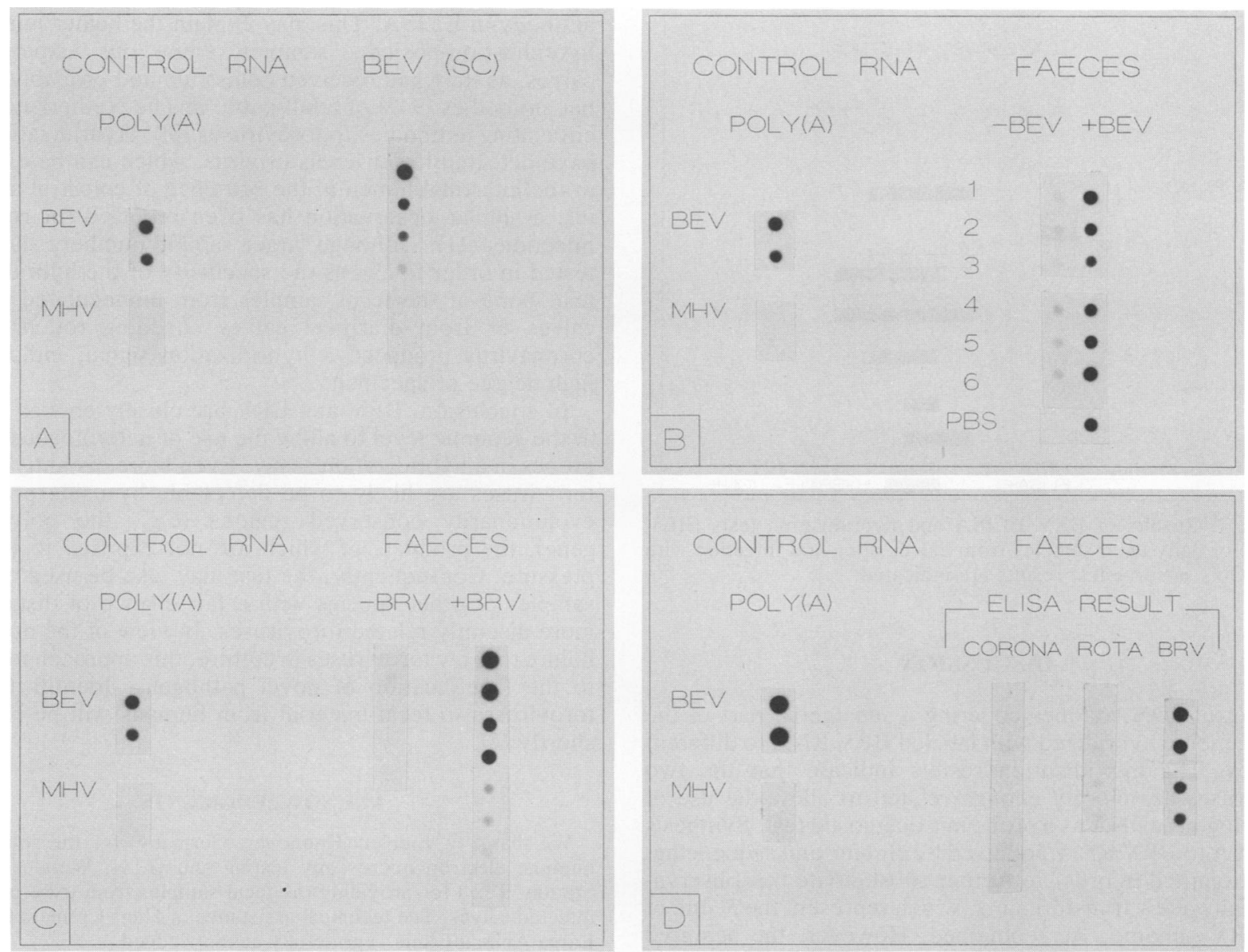

FIG. 3. Standardization of the dot hybridization assay for RNA detection by using a mixture of BEV cDNAs as a probe (clones 27,106 , 115, 120, 126, and 129 [Fig. 1]). Poly(A)-selected BEV and MHV RNAs were spotted (50 ng per spot) in each assay in duplicate as positive and negative controls. RNA extracted from the following samples was assayed. (A) Twofold dilutions of culture supernatant from BEV-infected EMS cells, starting at $10^{6} \mathrm{TCID}_{50}$ per sample. SC, Extracted by binding to silicon dioxide particles. (B) Fecal samples from six uninfected calves mixed with PBS $(-\mathrm{BEV})$ or with $5 \times 10^{5} \mathrm{TCID}_{50}$ of BEV (+BEV); extracts from PBS with or without BEV served as controls. (C) Serial twofold dilutions of feces from a calf after oral infection with BRV; the starting material contained $640 \mathrm{HAU}$. (D) Feces from diarrheal calves that had reacted in an ELISA for the detection of coronavirus, rotavirus, or BRV.

(16), showed weak hybridization and was therefore not used in the diagnostic test. Inserts from BEV cDNA clones 27 , $106,115,120,126$, and 129 , which are estimated to cover half of the genome (Fig. 2B), were mixed, labeled, and used as probes in the hybridization assays.

Establishment of the hybridization assay. Results of hybridizations of BEV cDNA with RNA that had been extracted by using guanidine isothiocyanate and silicon dioxide are shown in Fig. 3. Poly(A)-selected RNAs from BEV- and MHVinfected cells ( $50 \mathrm{ng}$ per spot) were used as positive and negative control preparations, respectively. Serial dilutions of culture supernatant from BEV-infected cells resulted in a positive hybridization signal with RNA extracted from $1.3 \times$ $10^{5}$ TCID $_{50}$ per spot (Fig. 3A). Six different ELISA-negative fecal samples were mixed with $5 \times 10^{5}$ TCID $_{50}$ of $\mathrm{BEV}$ or with phosphate-buffered saline (PBS); RNA was extracted and spotted, and hybridization with BEV cDNA was performed. Some background signal was observed in the negative fecal preparations, but a clear distinction could be made between the positive and negative samples (Fig. 3B). Subsequently, feces from BRV-infected GCs were serially diluted, extracted, and hybridized with BEV probes. Virus quantities equivalent to 20 hemagglutinating units (HAU) of the reference BRV preparation could be detected (Fig. 3C). We then extracted RNA from ELISA- and solid-phase immune electron microscopy-positive and -negative fecal samples from diarrheal calves that had been submitted to the Large Animal Clinic. Feces from diarrheal calves shedding rotavirus or coronavirus (as detected by ELISA) were included as specificity controls. Only BRV ELISA-positive samples reacted in the hybridization test (Fig. 3D).

Application of the hybridization assay as a diagnostic test. A total of 53 fecal samples from seven calves before and after infection with BRV, astrovirus, and cryptosporidia were assayed in parallel by ELISA and dot hybridization. In the hybridization test, distinct positive reactions were read after overnight exposure of the film. Some background signals were detected in a few preinoculation samples only after long exposure. Therefore, the hybridization tests were evaluated after short exposure only. By using ELISA, BRV was detected in 6 samples from three calves, whereas the hybridization test detected toroviral sequences in 16 samples from seven calves. Five samples reacted in both tests; one ELISA-positive sample was negative in the hybridization assay (Fig. 4). All 14 samples from three uninoculated control calves were negative in both tests. 


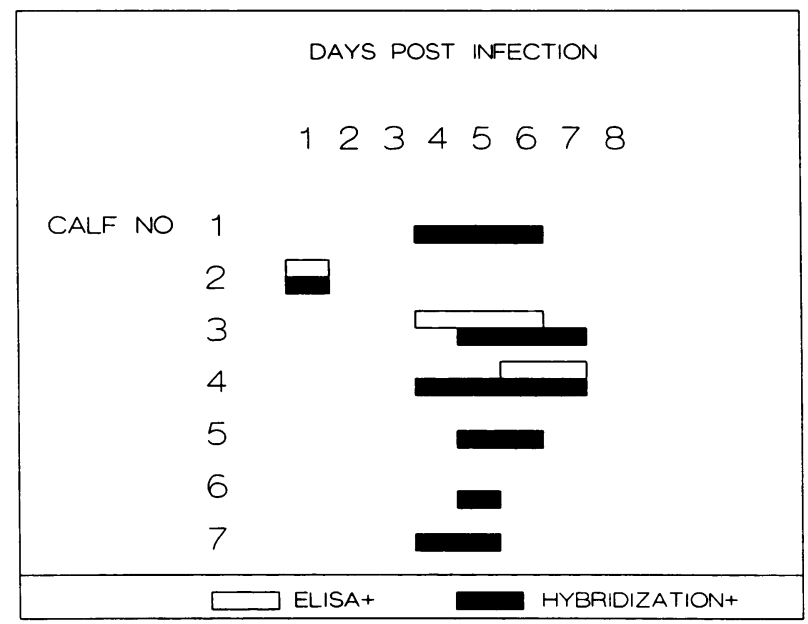

FIG. 4. Results of BRV ELISA and hybridization tests (BEV cDNA) of daily fecal samples from calves after oral infection with BRV. Only positive test results are indicated.

\section{DISCUSSION}

A set of cDNA clones covering a substantial part of the BEV genome hybridized with labeled BRV RNA to different degrees. The hybridization results indicate that the two toroviruses are closely enough related to allow the use of heterologous cDNA as a probe in a diagnostic test. Synthesis of cDNA to BRV RNA, followed by cloning and sequencing, was attempted in order to further substantiate this observation. Only a few transformants, which represent the $3^{\prime}$ end of the BRV genome, were obtained. However, the acquired sequence information showed that the $3^{\prime}$ ends of the genomes of both toroviruses are highly conserved (Fig. 1). The alignment includes the $3^{\prime}$ end of the $\mathrm{BEV} \mathrm{N}$ protein gene. At the amino acid level the similarity between the carboxy terminus of the BEV N protein and the corresponding BRV gene product was high, although this should be interpreted with caution until more sequence data have been obtained. However, the $\mathbf{N}$ protein is likely to be evolutionarily conserved since it is inaccessible to antibodies; less selection pressure may be expected to act on this protein compared with that acting on surface proteins (12). Indeed, low reactivity of BEV cDNA clone 109 , representing a part of the $P$ protein gene, with BRV RNA was observed. However, other clones containing $P$ gene sequences (clones 106 and 115) produced a stronger hybridization signal. Immunologic cross-reactions between BEV and BRV and between different BRV serotypes have been observed at the peplomer protein level (hemagglutination inhibition test, neutralization test) $(6,19)$, indicating that some regions of the $P$ protein gene and its product are conserved.

Results obtained with RNA extracted from fecal samples of recently BRV-infected calves showed that the hybridization assay is more sensitive than ELISA. By using the reference BRV preparation (GC58; stored at $-70^{\circ} \mathrm{C}$ for more than 5 years), hybridization was found to be slightly less sensitive (20 HAU detected) than ELISA, in which $10 \mathrm{HAU}$ of BRV can be detected. However, storage of this preparation has probably affected the quality of the genomic RNA; only cDNA clones with short inserts were obtained, whereas MHV RNA gave rise to clones with longer inserts.

Hybridization is unaffected by the presence of antibody in the feces, which can inhibit the binding of virus to capture antibody in ELISA. This may explain the higher number of hybridization-positive samples from our experimental calves, as they had received colostrum and probably maternal antibodies (94\% of adult cattle in The Netherlands have circulating antibodies to toroviruses [8]). Hybridization may have detected lower levels of virus, which can be expected in the intestinal lumen in the presence of colostral antibodies; a similar observation has been reported for rotavirus infections (11). Although larger sample numbers should be tested in order to assess the specificity of the hybridization test, none of the fecal samples from uninoculated control calves or from diarrheal calves shedding rotavirus and coronavirus produced a hybridization signal, indicating a high degree of specificity.

In conclusion, BEV and BRV are closely enough related at the genomic level to allow the use of heterologous cDNA probes in a hybridization assay. Even more distantly related toroviruses are likely to be detectable by using probes in evolutionarily conserved regions (e.g., the polymerase gene), the products of which are not exposed to immune pressure. Consequently, the test may also be used on fecal samples of other species with a fair chance of discovering more distantly related toroviruses. In view of the notorious failure to grow toroviruses in culture, this approach may lead to the identification of novel pathogens. Identification of toroviruses in fecal material from humans will be reported shortly (7).

\section{ACKNOWLEDGMENTS}

We thank E. Liebler (Hannover, Germany) for the solid-phase immune electron microscopy testing and G. N. Woode (College Station, Tex.) for providing the fecal samples from experimentally infected calves. The technical assistance of Hans Cremers from the Large Animal Clinic is gratefully acknowledged.

\section{REFERENCES}

1. Boom, R., C. J. A. Sol, M. M. M. Salimans, C. L. Jansen, P. M. E. Wertheim-van Dillen, and J. van der Noordaa. 1990. A rapid and simple method for purification of nucleic acids. $\mathbf{J}$. Clin. Microbiol. 28:495-503.

2. Feinberg, A. P., and B. Vogelstein. 1983. A technique for radiolabeling DNA restriction endonuclease fragments to high specific activity. Anal. Biochem. 132:6-13.

3. Gubler, U., and B. J. Hoffman. 1983. A simple and very efficient method for generating cDNA libraries. Gene 25:263-269.

4. Horzinek, M. C., T. H. Flewett, L. F. Saif, W. J. M. Spaan, M. Weiss, and G. N. Woode. 1987. A new family of vertebrate viruses: Toroviridae. Intervirology 27:17-24.

5. Koopmans, M., H. Cremers, G. N. Woode, and M. C. Horzinek. 1990. Breda virus (Toroviridae) infection and systemic antibody response in sentinel calves. Am. J. Vet. Res. 51:1443-1448.

6. Koopmans, M., J. Ederveen, G. N. Woode, and M. C. Horzinek. 1986. Surface proteins of Breda virus. Am. J. Vet. Res. 47:18961900.

7. Koopmans, M., F. Lamouliatte, and M. C. Horzinek. Unpublished data.

8. Koopmans, M., U. van den Boom, G. N. Woode, and M. C. Horzinek. 1989. Seroepidemiology of Breda virus in cattle using ELISA. Vet. Microbiol. 19:233-243.

9. Koopmans, M., L. van Wuijckhuise-Sjouke, Y. H. Schukken, H. Cremers, and M. C. Horzinek. Submitted for publication.

10. Maniatis, T., E. F. Fritsch, and J. Sambrook. 1982. Molecular cloning: a laboratory manual. Cold Spring Harbor Laboratory, Cold Spring Harbor, N.Y.

11. Saif, L. J., and K. L. Smith. 1985. Enteric viral infections of calves and passive immunity. J. Dairy Sci. 68:206-228.

12. Sekel, J. J., and D. C. Wiley. 1986. Antigenic variation in Hong Kong influenza virus hemagglutinins, p. 19-24. In T. H. Birbeck and C. W. Penn (ed.), Antigenic variation in disease. Society for 
General Microbiology, Oxford.

13. Snijder, E. J., J. A. den Boon, P. J. Bredenbeek, M. C. Horzinek, R. Rijnbrand, and W. J. M. Spaan. 1990. The carboxyl-terminal part of the putative Berne virus polymerase is expressed by ribosomal frameshifting and contains sequence motifs which indicate that toro- and coronaviruses are evolutionarily related. Nucleic Acids Res. 18:4535-4542.

14. Snijder, E. J., J. A. den Boon, G. M. G. M. Verjans, W. J. M. Spaan, and M. C. Horzinek. 1989. Identification and primary structure of the gene encoding the Berne virus nucleocapsid protein. J. Gen. Virol. 70:3363-3370.

15. Snijder, E. J., J. Ederveen, W. J. M. Spaan, M. Weiss, and M. C. Horzinek. 1989. Characterization of Berne virus genomic and messenger RNAs. J. Gen. Virol. 69:2135-2144.

16. Snijder, E. J., M. C. Horzinek, and W. J. M. Spaan. 1990. A 3 '-coterminal nested set of independently transcribed mRNAs is generated during Berne virus replication. J. Virol. 64:331-338.
17. Spaan, W. J. M., P. J. M. Rottier, M. C. Horzinek, and B. A. M. van der Zeijst. 1981. Isolation and identification of virus specific mRNAs in cells infected with mouse hepatitis virus (MHVA59). Virology 108:424-434.

18. van Berlo, M. F., P. J. M. Rottier, M. C. Horzinek, and B. A. M. van der Zeijst. 1986. Intracellular equine arteritis virus (EAV)specific RNAs contain common sequences. Virology 152:492496.

19. Weiss, M., F. Steck, and M. C. Horzinek. 1983. Purification and partial characterisation of a new enveloped RNA virus (Berne virus). J. Gen. Virol. 64:1849-1858.

20. Woode, G. N., D. E. Reed, P. L. Runnels, M. A. Herrig, and H. T. Hill. 1982. Studies with an unclassified virus isolated from diarrheic calves. Vet. Microbiol. 7:221-240.

21. Woode, G. N., L. J. Saif, M. Quesada, N. J. Winand, J. F. Pohlenz, and N. K. Gourley. 1985. Comparative studies on three isolates of Breda virus of calves. Am. J. Vet. Res. 46:1003-1010. 